\title{
NEAR-IR STUDIES OF RECURRENT NOVA V745 SCORPII DURING ITS 2014 OUTBURST
}

\author{
D. P. K. Banerjee ${ }^{1}$, Vishal Joshi ${ }^{1}$, V. Venkataraman ${ }^{1}$, N. M. AshoK ${ }^{1}$, G. H. Marion ${ }^{2,3}$, E. Y. HsiaO $^{4}$, And A. Raj ${ }^{1}$ \\ ${ }_{1}^{1}$ Physical Research Laboratory, Navrangpura, Ahmedabad, Gujarat 380009, India; orion@ prl.res.in \\ ${ }_{2}^{2}$ University of Texas at Austin, 1 University Station C1400, Austin, TX 78712-0259, USA \\ ${ }^{3}$ Harvard-Smithsonian Center for Astrophysics, 60 Garden Street, Cambridge, MA 02138, USA \\ ${ }^{4}$ Carnegie Observatories, Las Campanas Observatory, Colina El Pino, Casilla 601, Chile \\ Received 2014 February 25; accepted 2014 March 4; published 2014 March 28
}

\begin{abstract}
The recurrent nova V745 Scorpii underwent its third known outburst on 2014 February 6. Infrared monitoring of the eruption on an almost daily basis, starting from 1.3 days after discovery, shows the emergence of a powerful blast wave generated by the high velocity nova ejecta exceeding $4000 \mathrm{~km} \mathrm{~s}^{-1}$ plowing into its surrounding environment. The temperature of the shocked gas is raised to a high value exceeding $10^{8} \mathrm{~K}$ immediately after outburst commencement. The energetics of the outburst clearly surpass those of similar symbiotic systems like RS Oph and V407 Cyg which have giant secondaries. The shock does not show a free-expansion stage but rather shows a decelerative Sedov-Taylor phase from the beginning. Such strong shock fronts are known to be sites for $\gamma$-ray generation. V745 Sco is the latest nova, apart from five other known novae, to show $\gamma$-ray emission. It may be an important testbed to resolve the crucial question of whether or not all novae are generically $\gamma$-ray emitters by virtue of having a circumbinary reservoir of material that is shocked by the ejecta rather than $\gamma$-ray generation being restricted to only symbiotic systems with a shocked red giant (RG) wind. The lack of a free-expansion stage favors V745 Sco to have a density enhancement around the white dwarf (WD), above that contributed by a RG wind. Our analysis also suggests that the WD in V745 Sco is very massive and a potential progenitor for a future SN Ia explosion.
\end{abstract}

Key words: infrared: stars - novae, cataclysmic variables - stars: individual (V745 Scorpii) - techniques: spectroscopic

\section{INTRODUCTION}

The symbiotic recurrent nova (RN) V745 Scorpii experienced its third known outburst recently on 2014 February 6.694 UT (Stubbings 2014) with two earlier eruptions being recorded in 1937 and 1989. It is a relatively less well studied nova among the 10 currently known RNe (viz., T Pyx, IM Nor, CI Aql, V2487 Oph, U Sco, V394 CrA, T CrB, RS Oph, and V3890 Sgr) and belongs to the sub-class of RNe which have giant secondaries (viz., RS Oph, T CrB, and V3890 Sgr). The secondary has been classified as a giant of spectral type M6 III \pm 2 subclasses (Harrison et al. 1993; Anupama \& Mikolajewska 1999; Duerbeck 1989; Sekiguchi et al. 1990; Williams et al. 1991) with an orbital period of $510 \pm 20$ days (Schaefer 2009). V745 Sco is a very fast nova with $t_{2}$ and $t_{3}$ of 6.2 and 9 days, respectively, which is estimated to lie at a distance of $7.8 \pm 1.8 \mathrm{kpc}$ in the middle of the galactic bulge (Schaefer 2010). Optical studies of the 1989 eruption are documented in Sekiguchi et al. (1990) and Duerbeck (1989). The latter work shows the early spectroscopic evolution through a montage of eight spectra covering the period between $\sim 10$ to 40 days after outburst. Other spectroscopic studies include those by Williams et al. (2003) and Wagner et al. (1989). In the infrared (IR), Sekiguchi et al. (1990) recorded the light curves in the JHKL bands while a near-IR (NIR) spectrum at $\sim 70$ days after outburst was recorded by Harrison et al. (1993). The observational coverage of this RN is sparse and there are notably no early time IR spectra which record its evolution in the IR. This work, and another in preparation, should contribute to filling this gap.

We have been obtaining multi-epoch, photometric, and spectroscopic NIR observations in the $0.85-2.4 \mu \mathrm{m}$ region starting from 1.3 days after discovery. During the course of analysis, it was noticed that the emission lines were rapidly narrowing with time. This phenomenon is rarely seen and is indicative of decelerating matter associated with a shock which in turn can be associated with $\gamma$-ray generation (see below). The other similar instances where such a phenomenon was witnessed earlier were in RS Oph (Das et al. 2006) and V407 Cyg (Munari et al. 2011; Shore et al. 2011, 2012). Since the development of a strong shock in V745 Sco is a rare and significant phenomenon, we use part of our data covering only the $I$ and $J$ bands to analyze and study the implications of the event. In a follow-up paper, we will present a more detailed analysis using our complete $J H K$ spectroscopy and photometry, as in RS Oph (Banerjee et al. 2009).

The present 2014 outburst is being observed at all wavelengths from radio to the $\gamma$-ray regime (Rupen et al. 2014; Banerjee et al. 2014; Anupama et al. 2014; Page et al. 2014; Mukai et al. 2014; Luna et al. 2014; Rana et al. 2014; Cheung et al. 2014). Among the notable early results is the reported detection of $\gamma$-rays from the object (Cheung et al. 2014). This has important ramifications for the present study. $\gamma$-ray detections from novae are recent and few in number, and V745 Sco is only the sixth nova to be detected in $\gamma$-rays after V407 Cyg, Nova Sco 2012, Nova Mon 2012, Nova Del 2013, and Nova Cen 2013. All detections have been made by the Fermi LAT starting with the first detection in V407 Cyg in 2010 (Abdo et al. 2010). As per the current understanding, $\gamma$-rays from novae are generated by a diffusive acceleration mechanism as particles rebound back and forth across a shock front created by the nova's ejecta plowing into a pre-existing dense surrounding medium. Generically, it is the same principle that leads to the creation of high energy cosmic rays. A shock is thus an essential prerequisite for $\gamma$-ray generation, and hence it is very necessary to establish its presence unambiguously. V407 Cyg was the first $\gamma$-ray nova where the decelerating shock front was clearly detected (Munari et al. 2011). Unfortunately, during the 2006 RS Oph outburst no $\gamma$-ray observing facility, with comparable sensitivity as the 
Fermi telescope, was available. The generation mechanism of the $\gamma$-ray emission in novae is also under debate. For the shock to develop it is necessary to have a dense ambient medium into which the nova ejecta propagates and decelerates. In the case of V407 Cyg and RS oph, the pre-existing dense ambient medium is provided by the high mass loss from the secondary late-type giant star (the companion in V407 Cyg is a Mira variable). In contrast, for instance, in the case of Nova Mon 2012-another $\gamma$-ray nova-it is fairly certain that the companion is not a late-type giant (Munari et al. 2012), and hence cannot provide the dense ambient medium through copious mass loss. Thus there is uncertainty as to how $\gamma$-rays are indeed generated in novae systems. In this context, recent calculations show that a late-type giant's wind, solely by itself, may not be enough to create the requisite density enhancements necessary to explain the observed behavior of the $\gamma$-ray light curve. Additional sources of density enhancement in the form of a reservoir of circumbinary material around the white dwarf (WD) is perhaps needed (Martin \& Dubus 2013). This is a new point of departure from earlier thinking and, interestingly enough, similar arguments for pre-existing circumbinary material were already proposed by Williams (2013) from totally different considerations. In such a context, the eruption of V745 Sco is thus an important testbed for understanding unexplained aspects of $\gamma$-ray generation in novae.

\section{OBSERVATIONS}

NIR spectroscopy in the $0.85-2.4 \mu \mathrm{m}$ region at $R \sim 1000$ was carried out with the $1.2 \mathrm{~m}$ telescope of the Mount Abu Infrared Observatory (Banerjee \& Ashok 2012) using the Near-Infrared Camera/Spectrograph (NICS) equipped with a $1024 \times 1024$ HgCdTe Hawaii array. ${ }^{5}$ Spectra were recorded with the star dithered to two positions along the slit with one or more spectra being recorded in both of these positions. The coadded spectra in the respective dithered positions were subtracted from each other to remove sky and dark contributions. The spectra from these sky-subtracted images were extracted using IRAF tasks and wavelength calibrated using a combination of $\mathrm{OH}$ sky lines and telluric lines that register with the stellar spectra. To remove telluric lines from the target's spectra, it was ratioed with the spectra of a standard star from whose spectra the Hydrogen Paschen and Brackett absorption lines had been removed. The spectra were finally multiplied by a blackbody at the effective temperature of the standard stars SAO 186061 and SAO 209303. The log of the observations is given in Table 1 .

NIR spectra $(R \approx 6000, \lambda=0.8-2.5 \mu \mathrm{m})$ were obtained using the Folded Port Infrared Echellette (FIRE) spectrograph on the $6.5 \mathrm{~m}$ Magellan Baade Telescope (Simcoe et al. 2008). FIRE data are reduced using a custom IDL package (Simcoe et al. 2008) and additional procedures described by Hsiao et al. (2013).

\section{RESULTS AND DISCUSSION}

We present in Figure 1 the complete set of spectra in the $I J$ band between 0.85 and $1.35 \mu \mathrm{m}$ obtained from Mount Abu. The major lines are marked and it may be seen that most of the lines are of $\mathrm{H}$ and $\mathrm{He}$ with $\mathrm{He}$ I $1.0830 \mu \mathrm{m}$ overwhelming all other lines in strength. The NIR spectra are typical of the $\mathrm{He} / \mathrm{N}$ class of novae (Banerjee \& Ashok 2012) and are fairly similar

\footnotetext{
5 This paper includes data gathered with the $6.5 \mathrm{~m}$ Magellan Telescopes located at Las Campanas Observatory, Chile.
}

Table 1

Log of Observations

\begin{tabular}{lcccc}
\hline \hline UT & $\begin{array}{c}\text { Days after } \\
\text { Outburst }\end{array}$ & $\begin{array}{c}\text { Airmass } \\
\text { V745 Sco }\end{array}$ & $\begin{array}{c}\text { Telluric } \\
\text { Standard }\end{array}$ & $\begin{array}{c}\text { Airmass } \\
\text { Standard }\end{array}$ \\
\hline 2014 February 08.0 & 1.3 & 2.58 & SAO186061 & 2.05 \\
2014 February 09.0 & 2.3 & 3.29 & SAO186061 & 2.51 \\
2014 February 10.0 & 3.3 & 2.97 & SAO186061 & 2.03 \\
2014 February 11.0 & 4.3 & 3.12 & SAO186061 & 2.16 \\
2014 February 12.0 & 5.3 & 2.91 & SAO186061 & 2.08 \\
2014 February 13.0 & 6.3 & 2.73 & SAO186061 & 2.01 \\
2014 February 15.46 & 8.7 & 1.33 & HD163084 & 1.33 \\
2014 February 16.0 & 9.3 & 3.06 & SAO209303 & 3.13 \\
2014 February 17.0 & 10.3 & 3.06 & SAO209303 & 3.13 \\
2014 February 18.0 & 11.3 & 2.54 & SAO209303 & 2.53 \\
2014 February 19.0 & 12.3 & 3.05 & SAO209303 & 3.12 \\
2014 February 20.0 & 13.3 & 2.50 & SAO209303 & 2.54 \\
2014 February 22.0 & 15.3 & 2.33 & SAO209303 & 2.14 \\
\hline
\end{tabular}

Note. ${ }^{\text {a }}$ Observation from Magellan South. The remaining observations are from Mt. Abu. The outburst date is taken to be the discovery date viz. 2014 February 6.7 .

to those observed in RS Oph, except that the strength of Lyman beta fluoresced O I $1.1287 \mu \mathrm{m}$ line develops relatively much slower in V745 Sco. Unlike Duerbeck (1989), who detected coronal lines $\sim 10$ days after outburst, we do not see any coronal lines during the span of our observations. The highest excitation lines seen here are due to He II. The spectrum obtained from FIRE is showed in Figure 2. All the emission lines of Figure 1 are clearly seen here too but at higher resolution. The sequence of Brackett lines in the $H$ band between $\mathrm{Br} 10$ at $1.7362 \mu \mathrm{m}$ and $\mathrm{Br} 25$ at $1.4967 \mu \mathrm{m}$ is rather striking. A magnified view shows that mild first overtone $\mathrm{CO}$ emission at $2.29 \mu \mathrm{m}$ and beyond, arising from the secondary, had already begun to appear in the FIRE spectrum. The $\mathrm{CO}$ features became more pronounced with time as emission from the secondary becomes dominant. This is demonstrated in the bottom panel of Figure 1 which shows two $K$-band spectra: one at 1.3 days and the other 12.3 days after outburst-in the latter, the CO bands are clearly seen.

Figure 3 well illustrates the evolution of the profile of the $\operatorname{Pa} \beta 1.2818 \mu \mathrm{m}$ line, which was chosen because it is both a strong line and also unblended with other lines. Profile width measurements are therefore reliable. The observed profiles are composed of a broad component that is attributed to the nova ejecta on which is superposed a sharp and narrow component. The profiles are very similar to those seen in V407 Cyg in which the sharp component was attributed to the sudden ionization of a large fraction of the secondary's wind by the flash of energetic radiation produced by the thermonuclear event (Munari et al. 2011). A similar origin is proposed here too. What is striking is the rapid narrowing of the profiles with time. We decomposed each profile into two Gaussians representing the broad and narrow components, respectively, and measured the evolution of their FWHM values. A representative two-Gaussian fit to one of the profiles is shown in Figure 3 with the variation of the FWHMs with time is shown in the bottom panel. The right panel shows a similar evolution for the 2010 outburst of V407 Cyg from unpublished material not included in Munari et al. (2011). The shape and evolution of the profiles of both objects share a good similarity. For V745 Sco, the narrow component from the Mira wind does not show much variation. During the early stages, it is kinematically perturbed to some extent but its FWHM gradually tends to evolve toward its value observed at 


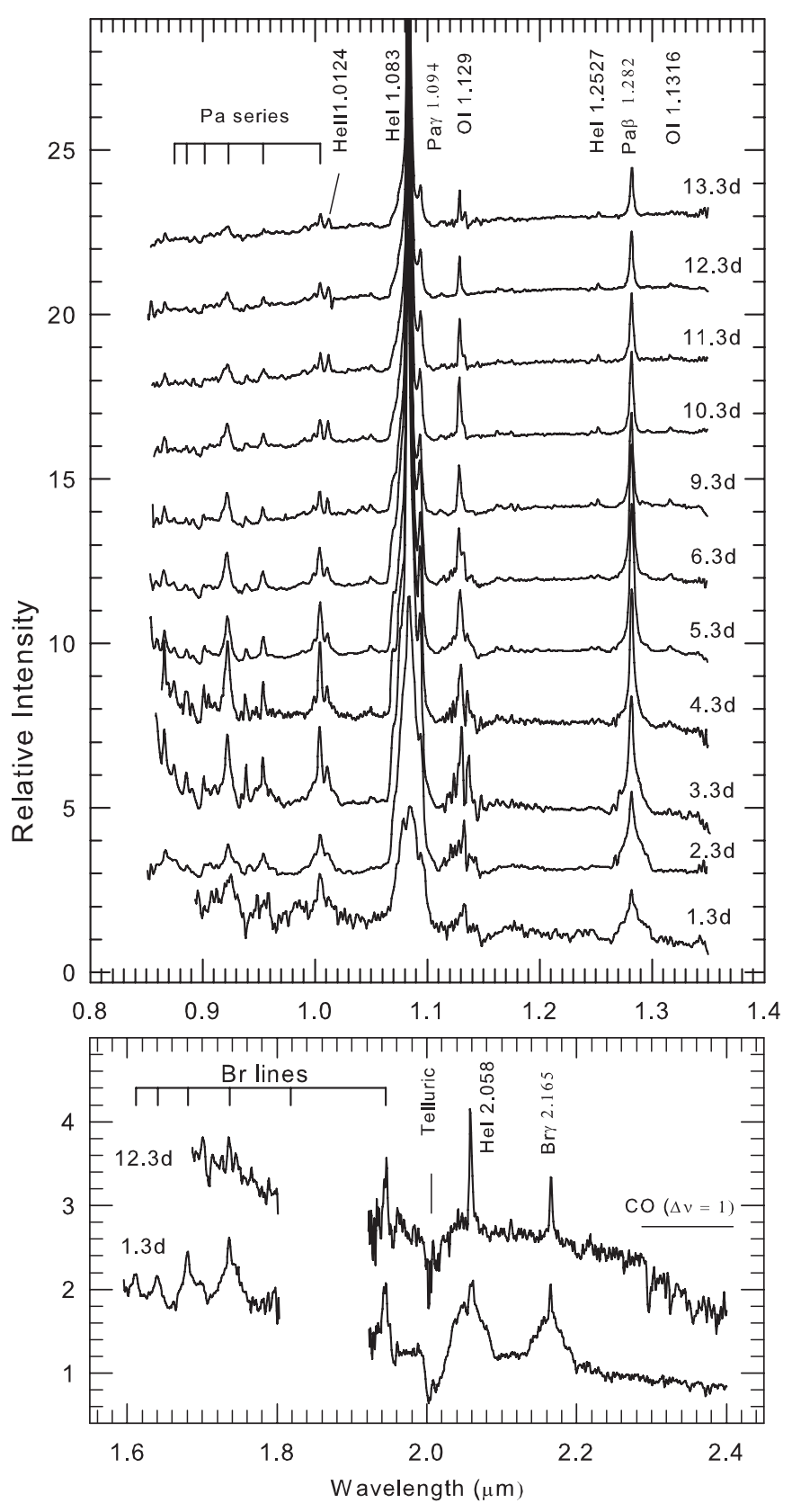

Figure 1. Temporal evolution of the $I J$ spectra (top panel) with days after outburst indicated. Representative HK spectra are shown (bottom panel) on 1.3 and 12.3 days after outburst. The prominent lines are marked.

quiescence. We consider the FWHM of the $\mathrm{H}$ lines in quiescence to be adequately represented by the FWHM of the $\mathrm{H} \beta$ line whose intrinsic width (corrected for instrumental broadening) is $\sim 450 \pm 15 \mathrm{~km} \mathrm{~s}^{-1}$ (U. Munari 2014, private communication) as presented in the high-resolution atlas of symbiotic stars by Munari \& Zwitter (2002). We discuss the evolution of the broad component in V745 Sco below.

The behavior of the shockwave as it propagates into the dense ambient medium surrounding the WD is usually divided into the following stages (e.g., Bode \& Kahn 1985). First is a freeexpansion or ejecta-dominated stage where the ejecta expands freely and the shock moves at a constant speed without being impeded by the surrounding medium. This phase generally extends to the time it takes for the swept-up mass to equal the

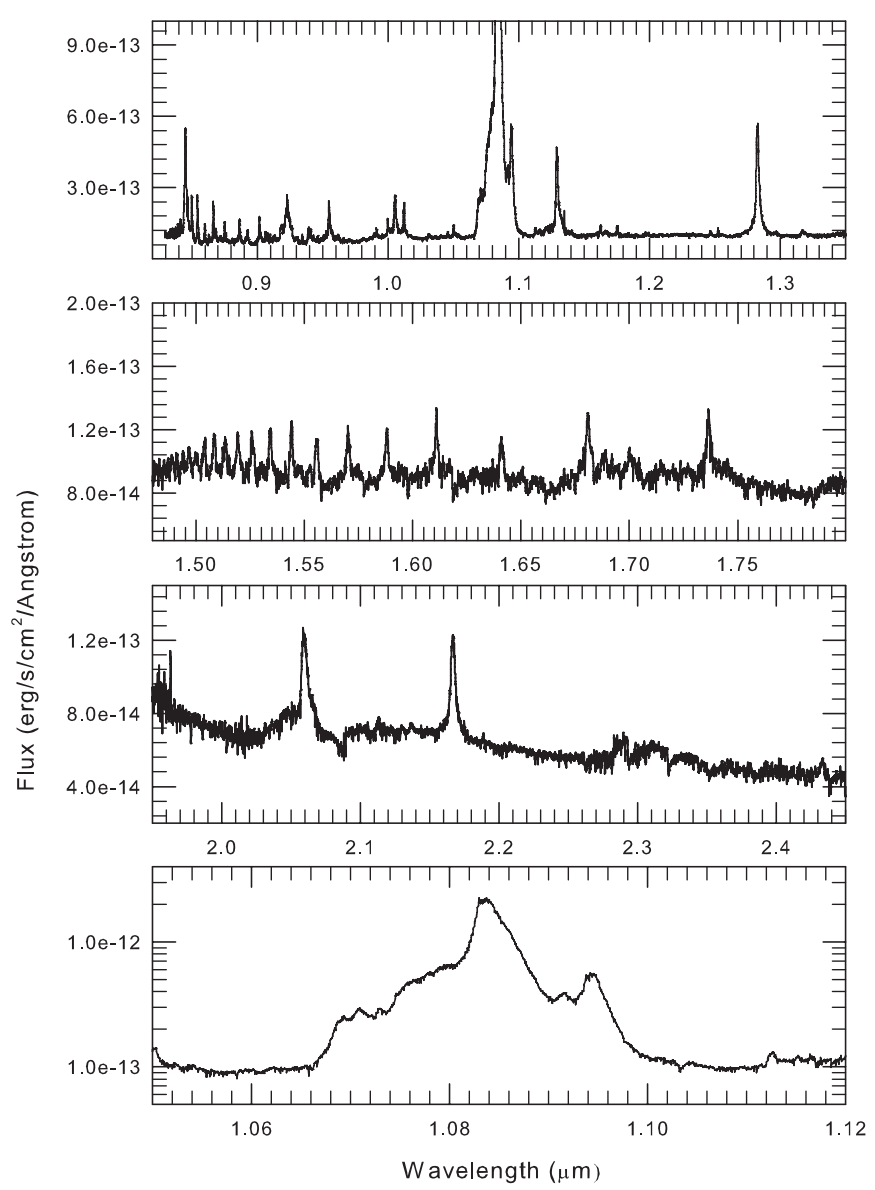

Figure 2. 0.8-2.5 $\mu \mathrm{m}$ FIRE spectrum on February 15.4 (8.7 days after outburst) is shown in the top three panels. The bottom panel shows an expanded view of the He I $1.0830 \mu \mathrm{m}$ line.

ejecta mass. The second phase is a Sedov-Taylor stage where the majority of the ejecta kinetic energy has been transferred to the swept-up ambient gas. This is an adiabatic phase since the shocked material is so hot that there is negligible cooling by radiation losses. During this phase, a deceleration is seen in the shock whose velocity $v$ versus time $t$ is expected to behave as $v \propto t^{-1 / 3}$, assuming a $r^{-2}$ dependence for the decrease in density of the wind. In phase 3 , the shocked material has cooled by radiation, and here the expected dependence of the shock velocity is $v \propto t^{-1 / 2}$. One may mention that the strong $\mathrm{X}$-ray blast wave seen during the 2006 outburst of RS Oph largely conformed to the above behavior (Sokoloski et al. 2006; Bode et al. 2006).

The free-expansion stage was not seen in V745 Sco, whose implications need to be understood. The intrinsic colors of the late M-type giant in V745 Sco are estimated to be $(J-H)_{0}$ $\sim 0.95$ and $(H-K)_{0} \sim 0.41$ using Two Micron All Sky Survey magnitudes of $J H K=10.04,8.85$, and 8.3, respectively, as quiescent values and correcting them using an adopted reddening value of $E(B-V)=0.70$ (Schlafly \& Finkbeiner 2011). A value of $E(B-V)>0.6$, for the estimated distance of $7.8 \mathrm{kpc}$ to $\mathrm{V} 745 \mathrm{Sco}$, is also supported from modeling of the galactic interstellar extinction by Marshall et al. (2006). On an IR color-color diagram (see Figures 4 and 6 of Whitelock \& Munari 1992), this places it among galactic bulge giants, a conclusion that was also reached by Sekiguchi et al. (1990). A comparison by Whitelock \& Munari (1992) of the IR characteristics of 

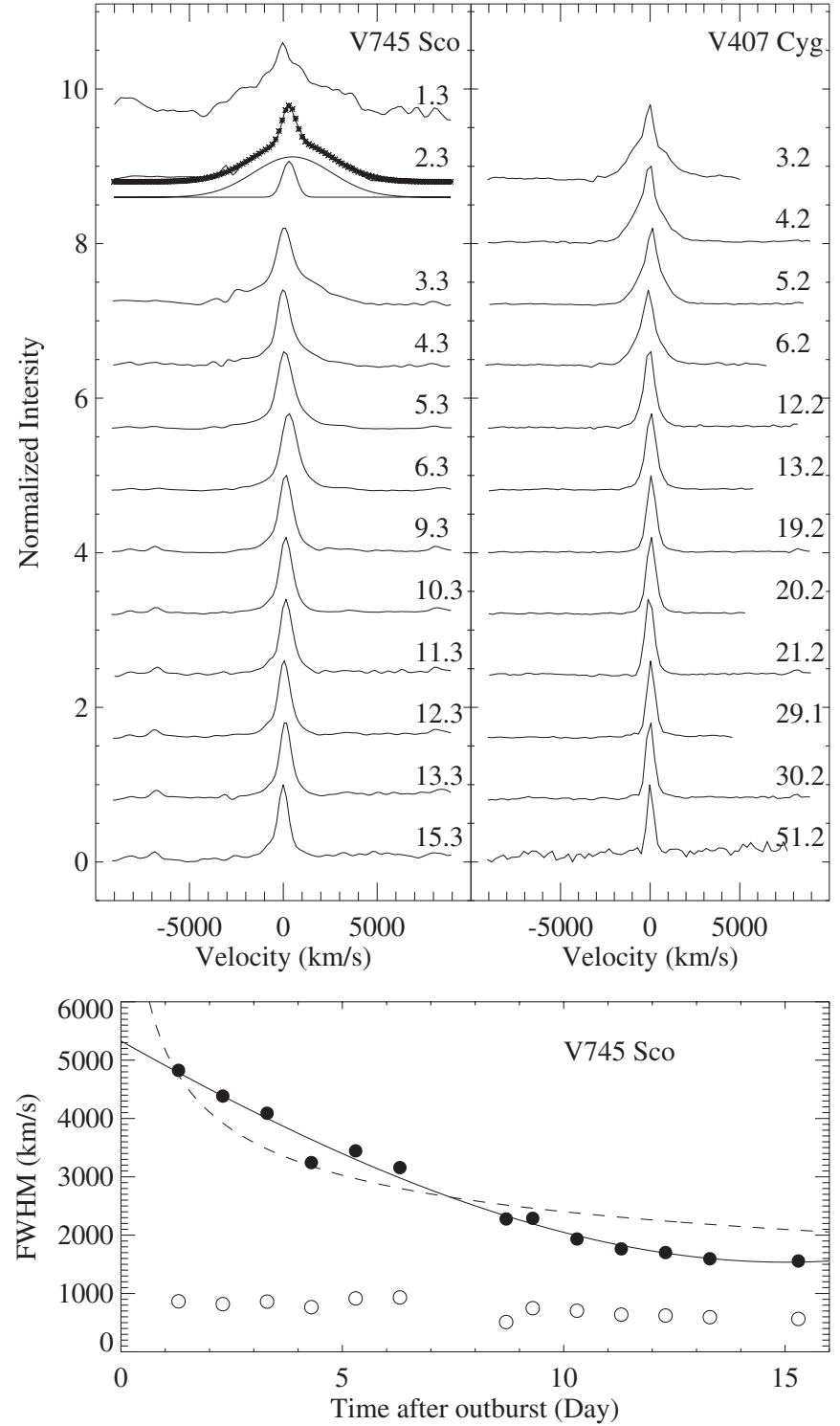

Figure 3. $\mathrm{Pa} \beta$ line profiles showing narrowing of the line width in V745 Sco (top left panel; details in Section 3). A sample two Gaussian fit to the profile 2.3 days after outburst is shown. The narrow and broad components are shown and their added sum is shown by star symbols. Similar plots for the line narrowing in the 2010 outburst of V407 Cyg are shown in the top right panel. The lower panel shows the temporal evolution of the FWHM of the broad and narrow components (dark and empty circles, respectively); the velocity decline is poorly fitted by a $t^{-1 / 3}$ law (dashed lines) but better approximated by a third degree polynomial (continuous line).

neighborhood $M$ giants, bulge $M$ giants, and the $M$ giants of $\mathrm{S}$-type symbiotic systems shows that the $\mathrm{M}$ giant secondaries in symbiotic systems are very similar to those in the bulge and are thus low-mass $\left(<1 M_{\odot}\right)$ objects. We adopt this as an upper limit for the mass of the secondary in V745 Sco. It is also likely that a significant fraction of all the symbiotic $M$ stars are actually asymptotic giant branch (AGB) stars rather than giant branch stars (Kniazev et al. 2009). This suggestion that symbiotics have AGB stars as mass donors would support the view that additional mass could be transferred through the stellar winds, above that transferred via Roche lobe overflow, since the winds from AGB stars are stronger than those from normal giants. We thus adopt a mass loss rate of around $10^{-7} M_{\odot} \mathrm{yr}^{-1}$. This is a reasonable value for an AGB star and is also in line with that chosen for V407 Cyg (Martin \& Dubus 2013).

Assuming a high mass for the WD (in the range 1.2-1.4 $M_{\odot}$ ), $M_{\mathrm{sec}} \sim 1 M_{\odot}$, and orbital period of 520 days, the separation between the binary components in V745 Sco is tightly constrained in the range 1.4-1.5 AU. In the elapsed time of 1.3 days between the onset of outburst and our first observation, the blast wave traveling at at over $4000 \mathrm{~km} \mathrm{~s}^{-1}$ will sweep up material within a radius of $3 \mathrm{AU}$ from the WD. The mass of this material is estimated to be $M_{\text {swept }}=0.7 \times 10^{-7} M_{\odot}$ assuming $(d \dot{M} / d t)_{\text {secondary }}=10^{-7} M_{\odot} \mathrm{yr}^{-1}$, a geometric $1 / r^{2}$ dilution in the density profile of the red giant (RG) wind, and a velocity of the RG wind of $10 \mathrm{~km} \mathrm{~s}^{-1}$. Increasing the wind velocity will reduce $M_{\text {swept }}$, while increasing $(d M / d t)_{\text {secondary }}$ will linearly increase it. However, for a reasonable physical choice of parameters, $M_{\text {swept }}$ appears to be constrained between $10^{-7}$ to $10^{-6} M_{\odot} . M_{\text {swept }}$ is estimated assuming the material between WD and secondary has no additional enhancements beyond that due to spherically symmetric wind from the secondary (see Figure 1 of Martin \& Dubus 2013). The free-expansion stage, if it ever occurred, had commenced and completed before our first observation made 1.3 days after discovery. The small value of matter swept out during these 1.3 days indicates one of two possibilities. First, the mass of the ejected matter $M_{\mathrm{ej}}$ in the outburst is small and of the order of $M_{\text {swept }}$. The small value of $M_{\mathrm{ej}}$ in turn would imply that the central WD is massive since the critical mass of the accreted envelope required to trigger a thermonuclear runaway is inversely proportional to the mass of the WD $\left(M_{\mathrm{acc}}=\left(4 \pi R_{\mathrm{WD}}{ }^{4} P_{\text {crit }} / G M_{\mathrm{WD}}\right)\right.$, where $P_{\text {crit }}=1020 \mathrm{dyne} \mathrm{cm}^{-2}$ for $M_{\mathrm{WD}}=1.4 M_{\odot}$; Truran \& Livio 1986). The second conclusion that can be drawn from a free-expansion stage that is either very short-lived ( $<1.3$ days) or absent is that the ejecta was very quickly impeded by additional material apart from the giant's wind. That is, the Martin \& Dubus (2013) hypothesis positing additional material enhancement, as applicable to V407 Cyg, is valid here too. Preliminary results from the $\gamma$-ray detection by Fermi-LAT data indicates that the detections with largest observed significances were on 2014 February 6 and 7 with no significant emission (within stipulated detection limits given) was detected in the subsequent days through the end of 2014 February 10 . The fact that the $\gamma$-rays peaked early, coincident with the optical outburst, strongly points to a dense circumbinary reservoir around the WD. The greatest difficulty that Martin $\&$ Dubus (2013) faced while reproducing the $\gamma$-ray light curve of V407 Cyg was in simulating the early peaking of the $\gamma$-ray emission using just an RG wind. To overcome this, they were forced to invoke the presence of dense additional matter close to the WD. It may be noted that the above argument does not rule out the possibility of a small ejecta mass (or equivalently a massive WD). Independent support for a high-mass WD is found in the extremely early turn-on of the super-soft X-ray phase at $\sim 3$ days after discovery (Page et al. 2014). This, coupled with the very high ejecta velocities observed, imply the presence of very low-mass ejecta and thereby a massive WD (Figure 6 of Schwarz Greg et al. 2011). V745 Sco thus could be a potential progenitor candidate for an SN Ia explosion by virtue of having a high-mass WD whose mass, as in other similar symbiotic systems like RS Oph, T CrB, and V3890 Sgr, is also suggested to be increasing after each outburst (Hachisu \& Kato 2001; Hachisu et al. 2007).

The adiabatic (decelerative) phase in Figure 2 deviates significantly from the $t^{-1 / 3}$ dependency, indicating that the shock is propagating into a wind which is not spherically symmetric. 
The reasons for this are twofold. The nova shell is expected to be slowed down more effectively in the parts moving in the direction of the RG due to the increasing density in that direction. In addition, as discussed above, there is additional material, most likely distributed over the equatorial plane. The combined effect of these is to make matter distribution around the WD's position anisotropic, and the shock front should thus rapidly become aspherical. Good support for this is offered by the detailed radio monitoring and modeling of the V407 Cyg outburst by Chomiuk et al. (2012; refer to their Figure 6). V745 Sco was observed 10 days after the optical discovery on February16 with NUSTAR showing a luminous hard X-ray source whose spectrum could be modeled by a plasma in collisional ionization equilibrium at $k T=2.6 \mathrm{keV}$, or equivalently $3.02 \times 10^{7} \mathrm{~K}$ (Rana et al. 2014). This is consistent with what we observe. For a strong shock, the post-shock temperature $T_{s}$ is given by $T_{s}=\left(3 \bar{m} v^{2} / 16 k\right)$, where $k$ is the Boltzmann constant and $\bar{m}=10^{-24} \mathrm{~g}$ is the mean particle mass including electrons (Bode et al. 2006). On day 10.3, we measure $v=$ $1930 \mathrm{~km} \mathrm{~s}^{-1}$ equivalent to a temperature of $5.05 \times 10^{7} \mathrm{~K}$, which agrees satisfactorily with the X-ray result. Extending the calculations, the gas must have been heated to extremely high temperatures exceeding $1 \times 10^{8} \mathrm{~K}$ at 1.3 days when the FWHM was $4825 \mathrm{~km} \mathrm{~s}^{-1}$. In comparison, in RS Oph whose evolution was very well documented, a $\mathrm{Pa} \beta$ FWHM of $3066 \mathrm{~km} \mathrm{~s}^{-1}$ on 1.16 day was measured (Das et al. 2006) and for the same line in V407 Cyg we obtained a value $1862 \mathrm{~km} \mathrm{~s}^{-1}$ on 2010 March 13 (3.2 days after outburst; Munari et al. (2011) measured a FWHM of $2760 \mathrm{~km} \mathrm{~s}^{-1}$ on day +2.3 from the $\mathrm{H} \alpha$ profile). Clearly, the outburst of V745 Sco is extremely powerful, an aspect that needs to be emphasized and which was not established from its earlier outbursts. Its energetics overshadow even those of RS Oph and V407 Cyg.

Research at PRL is supported by the Department of Space, Government of India. G.H.M. thanks D. Osip, P. Palunas, Y. Beletsky, and the engineering group at the Las Campanas Observatory for their support of observations. The CfA Supernova Program is supported by NSF grant AST-1211196 to the Harvard College Observatory. We thank the reviewer Professor Ulisse Munari for helpful comments.

\section{REFERENCES}

Abdo, A. A., Ackermann, M., Ajello, M., et al. 2010, Sci, 329, 817 Anupama, G. C., \& Mikolajewska, J. 1999, A\&A, 344, 177

Anupama, G. C., Selvakumar, G., Moorthy, V., Velu, C., \& Dinakaran, N. 2014, ATel, 5871, 1

Banerjee, D. P. K., \& Ashok, N. M. 2012, BASI, 40, 243

Banerjee, D. P. K., Das, R. K., \& Ashok, N. M. 2009, MNRAS, 399, 357

Banerjee, D. P. K., Venkataraman, V., Joshi, V., \& Ashok, N. M. 2014, ATel, 5865,1

Bode, M. F., \& Kahn, F. 1985, MNRAS, 217, 205

Bode, M. F., O’Brien, T. J., Osborne, J. P., et al. 2006, ApJ, 652, 629

Cheung, C. C., Jean, P., \& Shore, S. N. 2014, ATel, 5879, 1

Das, R. K., Banerjee, D. P. K., \& Ashok, N. M. 2006, ApJL, 653, L141

Chomiuk, L., Krauss, M. I., Rupen, M. P., et al. 2012, ApJ, 761, 173

Duerbeck, H. W. 1989, Msngr, 58, 34

Hachisu, I., \& Kato, M. 2001, ApJ, 558, 323

Hachisu, I., Kato, M., \& Luna, G. J. M. 2007, ApJL, 659, L153

Harrison, T. E., Johnson, J. J., \& Spyromilio, J. 1993, AJ, 105, 320

Hsiao, E. Y., Marion, G. H., Phillips, M. M., et al. 2013, ApJ, 766, 72

Kniazev, A. Y., Vaisanen, P., Whitelock, P. A., et al. 2009, MNRAS, 395, 1121

Luna, G. J. M., Pillitteri, I., Mukai, K., \& Sokoloski, J. L. 2014, ATel, 5881, 1

Marshall, D. J., Robin, A. C., Reyle, C., et al. 2006, A\&A, 453, 635

Martin, P., \& Dubus, G. 2013, A\&A, 551, 37

Mukai, K., Page, K. L., Osborne, J. P., \& Nelson, T. 2014, ATel, 5811, 1

Munari, U., Dallaporta, S., Castellani, F., \& Valisa, P. 2012, MNRAS, 435, 771

Munari, U., Joshi, V., Ashok, N. M., et al. 2011, MNRAS, 410, L52

Munari, U., \& Zwitter, T. 2002, A\&A, 383, 188

Page, K. L., Osborne, J. P., Beardmore, A. P., \& Mukai, K. 2014, ATel, 5870, 1

Rana, V., Orio, M., Sokoloski, J., \& Harrison, F. 2014, ATel, 5913, 1

Rupen, M. P., Mioduszewski, A. J., Chomiuk, L., et al. 2014, ATel, 5874, 1

Schaefer, B. E. 2009, ApJ, 697, 721

Schaefer, B. E. 2010, ApJS, 187, 275

Schlafly, E., \& Finkbeiner, D. P. 2011, ApJ, 737, 103

Schwarz Greg, J., Ness, J.-U., Osborne, J. P., et al. 2011, ApJS, 197, 31

Sekiguchi, K., Whitelock, P. A., Feast, M. W., et al. 1990, MNRAS, 246, 78

Shore, S. N., Wahlgren, G. M., Augusteijn, T., et al. 2011, A\&A, 527, 98

Shore, S. N., Wahlgren, G. M., Augusteijn, T., et al. 2012, A\&A, 540, 55

Simcoe, R. A., Burgasser, A. J., Bernstein, R. A., et al. 2008, Proc. SPIE, 7014 $70140 \mathrm{U}$

Sokoloski, L. J., Luna, G. J. M., Mukai, K., et al. 2006, Natur, 442, 276

Stubbings, R. 2014, CBET, 3803, 1

Truran, J. W., \& Livio, M. 1986, ApJ, 308, 721

Wagner, R. M., Bertram, R., Starrfield, S., \& Shore, S. 1989, IAUC, 4822, 1

Whitelock, P. A., \& Munari, U. 1992, A\&A, 255, 171

Williams, R. E. 2013, AJ, 146, 55

Williams, R. E., Hamuy, M., Phillips, M. M., et al. 1991, ApJ, 376, 721

Williams, R. E., Hamuy, M., Phillips, M. M., et al. 2003, JAD, 9, 3 